\title{
A novel method to accurately align the laser sheet for planar and stereoscopic PIV
}

\author{
M. Shehzad*, S. Lawrence, C. Atkinson, J. Soria
}

Laboratory for Turbulence Research in Aerospace and Combustion (LTRAC), Mechanical and Aerospace Engineering, Monash University (Clayton Campus), Melbourne 3800 VIC, Australia *Muhammad.shehzad@monash.edu

\begin{abstract}
Several techniques including two-dimensional (2D) and three-dimensional (3D) calibration are used for the calibration of two-component two-dimensional (2C-2D) particle image velocimetry (PIV) and three-component two-dimensional (3C-2D) stereoscopic PIV (SPIV) systems. A major requirement of these techniques is to keep the calibration target exactly at the position of the laser sheet within the field of view (FOV), which is very difficult to achieve (Raffel et al., 2018). In 3C-2D SPIV, several methods offer different correction schemes based on the disparity between the FOV of two stereo cameras produced due to misalignment, to account for the misalignment error. These techniques adjust the calibration or the measured displacement field in different ways to reduce the error which may introduce an unintended error in the measurement position and/or velocity such as a bias in the measured three-component 3C displacements. This paper introduces a novel method to align the laser sheet with the calibration target so that the uncertainty in displacement measurements is minimal. Ideally, it should be of the order of the uncertainty associated with PIV measurement so that no ad hoc post-correction scheme is required.

The key to this novel method is to position the laser sheet and align it in parallel to the calibration target. For this purpose, the calibration target is fixed in a frame that has the capability to check the parallelism of the laser sheet relative to the target. This is confirmed by shaping the frame in a hollow rectangular form which lets the light sheet pass through its one end which has a $1 \mathrm{~mm}$ wide slit and detects it on the opposite end using three collinear equally-distant photo-detectors $(\mathrm{PD}\{1,2,3\})$ at $Y_{h}=[+62,0,-62][\mathrm{mm}]$ where $Y_{h}=0$ represents the location of the middle photo-detector. These PDs, connected to a multi-channel oscilloscope, detect the intensity of laser light falling on them through $1 \mathrm{~mm}$ holes. The intensity measurement is indirect, in the form of a voltage (unit: millivolt, $m V$ ). The rectangular frame which holds the calibration target in place is referred to as a calibration target mount and is the main component of the alignment setup. A schematic of the set-up is shown in figure 1(a) along with the calibration target and three photo-detectors.

The ability to manoeuvre the laser sheet is achieved using at least one $90^{\circ}$ laser mirror mount which is equipped with two adjusting screws to translate the reflected laser beam in the lateral and vertical directions before it is converted to a laser sheet. After the mirror, a rotation stage on which the sheet forming lens is fixed is used to rotate the laser sheet with an accuracy of $0.014^{\circ}$. The laser sheet is considered aligned with the target mount when maximum values of intensity are measured at all PDs and the intensity values at PD1 (at $Y_{h}=+62 \mathrm{~mm}$ ) and PD3 (at $Y_{h}=-62 \mathrm{~mm}$ ) are very close to each other (within $2 \%$ of the maximum intensity measured at PD2). This position of the laser sheet is taken as a reference position, $\Psi_{0}$ where $\Psi$ is the angle of the laser sheet rotation with respect to the calibration target mount. Rotating the laser sheet slightly about the expansion axis by increments of $0.1^{\circ}$ with respect to the reference position while keeping the pivot point of the sheet rotation at the middle hole will decrease the intensity values measured at PD1 and PD3 while at $\mathrm{PD} 2$, the intensity would remain constant. If the rotation is greater than $0.92^{\circ}$, almost no intensity is expected to be detected at PD1 and PD3. This is evident in figure 1(b) which shows the intensity values at PD1, PD2 and PD3 relative to the maximum intensity at PD2 when $\Psi=\Psi_{o}$, for the sheet rotation from $\Psi_{o}$ to $\Psi=0.9^{\circ}$. Moving the laser sheet slightly along $Z$ axis will decrease the intensities of all PDs while moving along a positive $Y$ axis will decrease intensities at PD2 and PD3 and increase at PD1. This sensitivity method assists in choosing the optimal laser sheet position relative to the calibration target and is therefore considered as the position of the light sheet where it is parallel
\end{abstract}


to the calibration target. Keeping the laser sheet fixed now and traversing the target mount by $Z=8.5 \pm 0.01 \mathrm{~mm}$ using a micrometre translation stage will bring it to the middle of the laser sheet.

An experiment has been performed to validate the application of the novel alignment method in 3C-2D SPIV. A transparent box containing randomly dispersed micro-particles is illuminated by the aligned laser sheet and translated by several sets of the known true displacements $T_{X}, T_{Y}$ and $T_{Z}$ in $X, Y$ and $Z$ axes. The ranges of the true displacements applied are: $T_{X}=[0.0,0.5] \mathrm{mm}, T_{Y}=[0.0,0.38] \mathrm{mm}$, $T_{X}=[-0.5,0.5] \mathrm{mm}$. Particle images are recorded at every set of translations using two stereo cameras (PCO pixelfly). These cameras are mounted on the Scheimpflug adapters and use Micro-NIKKOR lenses of the focal length of $55 \mathrm{~mm}$, with their optical axes at the nominal angles of $+45^{\circ}$ and $-45^{\circ}$ to $Z$ axis in the $X-Z$ plane. For the calibration, the images of the calibration target are recorded at $\Psi_{o}$ and $Z=[-0.5,0.0,0.5] \pm 0.01 \mathrm{~mm}$. The Soloff method (Soloff et al., 1997) of 3D calibration is used to map the 3D object space to the $2 \mathrm{D}$ image plane. The particle images are then dewarped using the computed 3D mapping functions. The dewarped particle images are individually paired with the one at $T_{X}, T_{Y}, T_{Z}=[0,0,0]$. Multigrid/multipass $2 \mathrm{C}-2 \mathrm{D}$ digital PIV cross-correlation analysis (Soria, 1996) using an in-house software is performed on the image pairs of both cameras to compute the $\left(u_{1}, v_{1}\right)$ and $\left(u_{2}, v_{2}\right)$ displacement vectors with respect to $X, Y, Z=(0,0,0)$. The $3 \mathrm{C}$ displacement vector ( $U V W$ ) is computed from the $2 \mathrm{C}$ displacement vectors using the $3 \mathrm{C}$ reconstruction technique of Willert (1997). The measured 3C displacements are then compared with the true displacements $T_{X}, T_{Y}$ and $T_{Z}$ to determine the residual error. A maximum uncertainty $7.6 \mu \mathrm{m}$ is found in the $3 \mathrm{C}-2 \mathrm{D}$ SPIV measurements. This is below the accuracy of the translation stages $(10 \mu \mathrm{m})$ which were used to apply the true displacement. Also, it is comparable to the uncertainty of the gther methods used to correct the misalignment error e.g. the self-calibration method (Wieneke, 2005). This shows that the new method provides a viable alternative to avoid or reduce the misalignment error without the risk of introducing bias into the results.

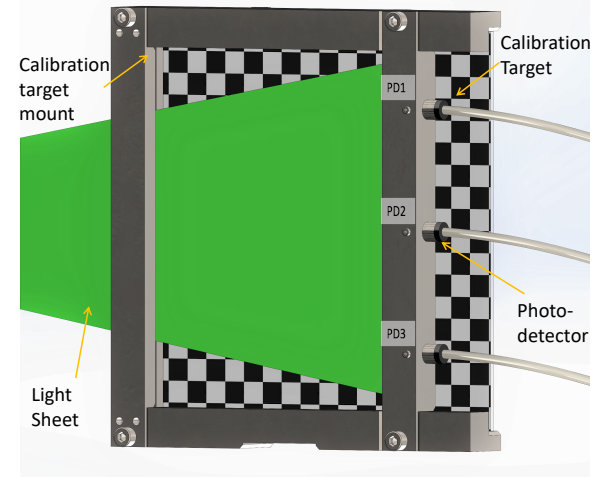

(a)

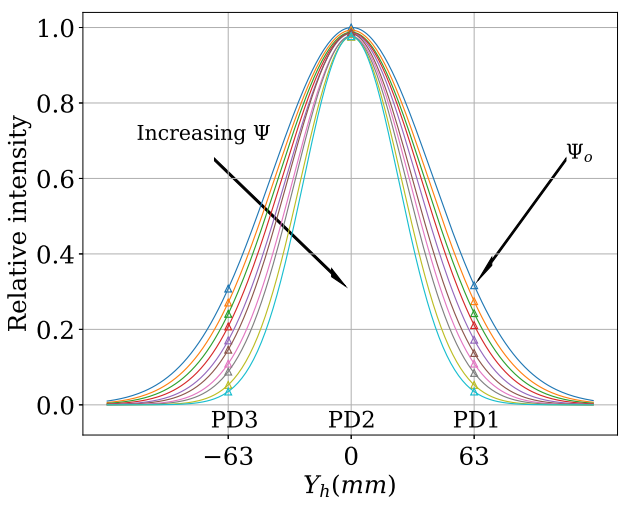

(b)

Figure 1: (a) Parametric view of the laser sheet alignment setup (b) Intensity values at PD1, PD2 and PD3 relative to the maximum intensity at PD2 when $\Psi=\Psi_{o}$, for the gradual rotation of laser sheet with increments of $0.1^{\circ}$. The angle of rotation is increasing in the direction of the black arrow.

\section{Acknowledgements}

The authors would like to acknowledge the support of the Australian Government for this research through an Australian Research Council Discovery grant. The authors also acknowledge the provision of the computational resources on Massive through National Computational Merit Allocation Scheme (NCMAS). Muhammad Shehzad also acknowledges the Punjab Educational Endowment Fund (PEEF), Punjab, Pakistan for funding his PhD research. Sean Lawrence gratefully acknowledges the financial support of the Maritime Division of the Defence Science and Technology Group. C. Atkinson was supported by the ARC Discovery Early Career Researcher Award (DECRA) fellowship. 


\section{References}

Raffel M, Willert CE, Scarano F, Kähler CJ, Wereley ST, and Kompenhans J (2018) Particle image velocimetry: a practical guide. Springer

Soloff SM, Adrian RJ, and Liu ZC (1997) Distortion compensation for generalized stereoscopic particle image velocimetry. Measurement science and technology 8:1441

Soria J (1996) An investigation of the near wake of a circular cylinder using a video-based digital cross-correlation particle image velocimetry technique. Experimental Thermal and Fluid Science $12: 221-233$

Wieneke B (2005) Stereo-piv using self-calibration on particle images. Experiments in fluids 39:267280

Willert C (1997) Stereoscopic digital particle image velocimetry for application in wind tunnel flows. Measurement science and technology 8:1465 